gastroenterologist. The objectives of this service evaluation were to assess satisfaction rates with the DLC and establish GFD compliance rates of patients attending the DLC.

Subjects and Methods Patients who had attended the DLC in the previous year were invited to partake in the service evaluation. The method was two online questionnaires. The satisfaction questionnaire had previously been utilised in the same centre to assess satisfaction with the service prior to the change to a DLC service. The dietary compliance questionnaire had previously been utilised in another paediatric study.

Results The patient population response rate was $40 \%$ $(n=28) .61 \%$ of respondents were 'extremely likely' to recommend the DLC to friends and family if similar care was needed. $61 \%$ of respondents were 'fully compliant', 32\% of respondents were 'compliant with errors' and 7\% of respondents were 'non-compliant' with a GFD. 79\% were 'fully compliant' in the age-group 3-11 years compared to $43 \%$ in the age-group 12-18 years. Chi-square analysis showed this difference was approaching statistical significance $(p=0.053)$. The responses to the question 'My child eats food labelled 'May contain traces of gluten or wheat'" stratified by the two agegroups showed that $86 \%$ of respondents in the age-group 311 years responded 'no' whereas 50\% of the respondents in the age-group 12-18 years responded 'no' to this question. Chi-square analysis showed that this difference reached statistical significance $(p=0.043)$.

Summary and Conclusion A comparison between the satisfaction survey results performed prior to the service change shows that there is an increased proportion of respondents from the DLC service who were 'extremely likely' to 'recommend the service to a friend or family member' (61\%) compared to the previous service (38\%). During the two year period since the DLC service has been in place, two patients from 70 patients who are reviewed in the DLC required referral to the gastroenterologist. Significant financial savings are associated with a DLC compared with the previous service. The compliance rates of the overall population group and the lower compliance rates in the adolescent sub-group found are in accordance with the available literature. The questionnaire was able to identify some areas where adolescents were falling down in their compliance to a GFD and this informed the development of an online education resource.

\section{P06 A SINGLE CENTRE DESCRIPTION OF IBD PATIENTS WITH NEGATIVE FAECAL CALPROTECTIN AT DIAGNOSIS}

Harween Dogra, Vinod Kolimarala, Bukunola Kukoyi, Babu Vadamalayan. King's College Hospital

\subsection{6/flgastro-2021-bspghan.17}

Introduction In the past 10 years faecal calprotectin has been increasingly used in Paediatrics, as in adults, to screen for inflammatory bowel disease (IBD). Faecal calprotectin is a calcium and zinc binding protein expressed by neutrophils and can be detected in stool when there is infiltration of the mucosa with neutrophils, as in IBD. Discussion around diagnostic values in Paediatrics have shown young children can have falsely elevated faecal calprotectin and studies have suggested values over 200 , or some over 800 , as a positive indication to investigate further for IBD. However, there are limited descriptions of IBD patients with negative faecal calprotectin and how this may influence investigation and management.
Methods We looked at retrospective data 20011-2020 of our IBD case load to identify how many had a negative/low faecal calprotectin (low FC) at diagnosis, this limit was set at $<80$, which is the current value for our hospital laboratory. Positive/raised faecal calprotectin (high FC) was any value $>80$. Our local database was used to identify IBD patients and the hospital electronic patient records system was searched for faecal calprotectin values at time of diagnosis (pre-treatment).

Results A total of 198 patients were diagnosed with IBD in the time frame investigated. $17(9 \%)$ patients had a negative faecal calprotectin at diagnosis, $118(60 \%)$ had a positive value, and 63 (32\%) had no documented value (figure 1). The difference in FC values was significantly different between the low FC and high FC group, $\mathrm{p}<0.0001$ (Mann-Whitney test, figure 3$)$. The median age at diagnosis was 14 yrs (2-16 yo) in the low FC group, and 12 yrs (1-17 yo) in the high FC group (figure 2). The distribution of IBD diagnosis in the

Total IBD patients 198 I

Low FC 17 High FC 118 Not documented 63

Abstract P06 Figure 1 Total of 198 IBD patient reviewed, 63 with no documented FC (faecal calprotectin), 17 with low/normal FC and 118 with high/raised FC

\begin{tabular}{|l|l|l|}
\hline & Low FC & High FC \\
\hline Age, median (range) & $14(2-16)$ & $12(1-17)$ \\
\hline Crohn's Disease & 5 & 61 \\
\hline Ulcerative colitis & 6 & 43 \\
\hline IBD-U & 6 & 14 \\
\hline
\end{tabular}

Abstract P06 Figure 2 Median age of patients with low/normal FC (faecal calprotectin) is 14yo, 5 patients with Crohn's disease, 6 patients with Ulcerative colitis, 6 patients with IBD-U. Median age of patients with high/raised FC is 12yo, 61 patients with Crohn's disease, 43 patients with Ulcerative colitis, 14 patients with IBD-U

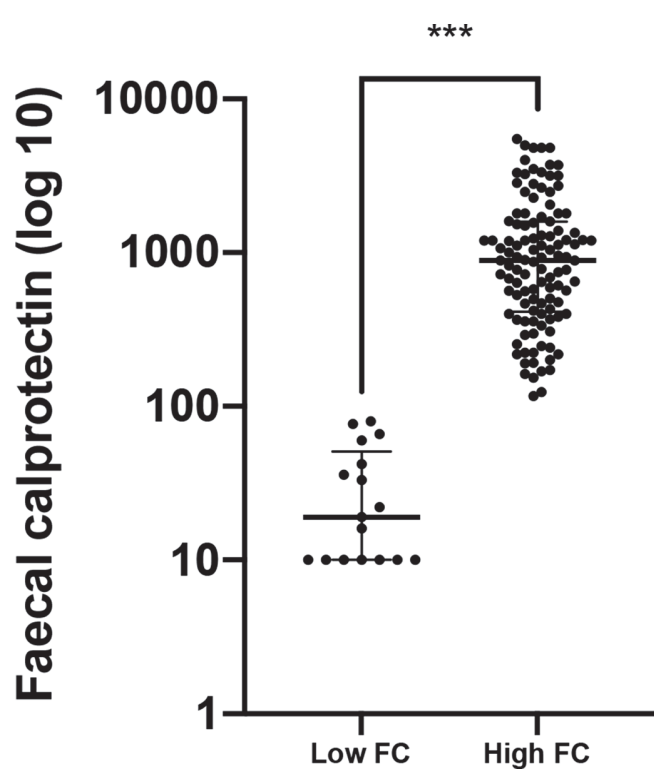

Abstract P06 Figure 3 There was a significant difference in FC (Faecal calprotectin) values between the normal/low FC and raised/high FC groups, ${ }^{* * *} p<0.0001$ (Mann-Whitney test) 
low FC compared to the high FC groups was Crohn's Disease $30 \%$ v 52\%, Ulcerative colitis $35 \%$ v 36\%, and IBD-U $30 \%$ $\mathrm{v} 12 \%$. The low and high FC values were significantly

\section{Crohn's Dlsease}

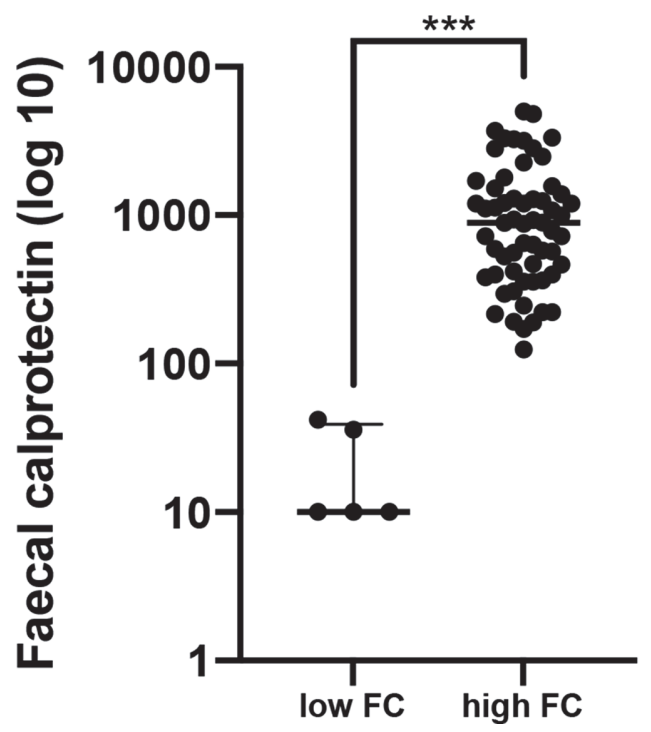

Abstract P06 Figure 4 In patients with Crohn's Disease, there was a significant difference in FC (Faecal calprotectin) values between the normal/low FC and raised/high FC groups, *** $p<0.0001$ (MannWhitney test)

\section{IBD-Unclassified}

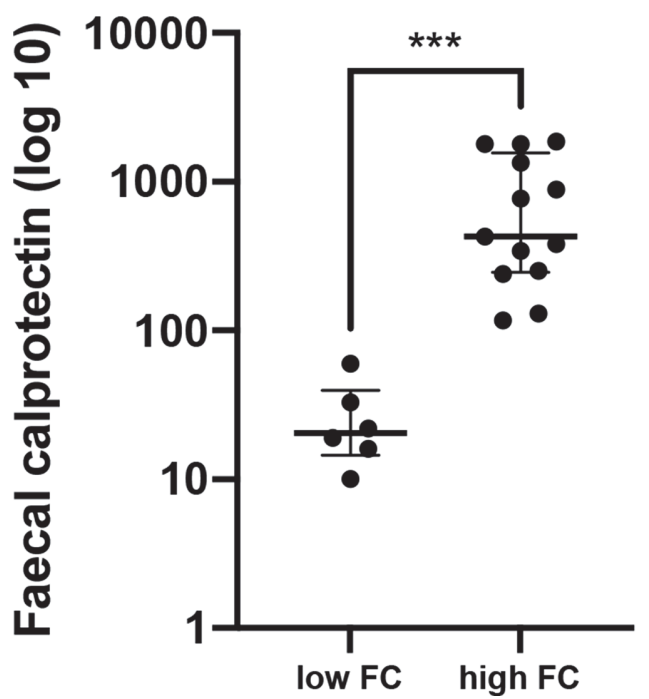

Abstract P06 Figure 6 In patients with IBD-Unclassified, there was a significant difference in FC (Faecal calprotectin) values between the normal/low FC and raised/high FC groups, *** $\mathrm{p}<0.0001$ (MannWhitney test)

different $(\mathrm{p}<0.0001)$ in all diagnostic sub-groups (figures 4,5 and 6).

Conclusion A small but significant percentage of our IBD patients had a negative faecal calprotectin at diagnosis. The majority did, during disease monitoring, develop a raised faecal calprotectin. Due to variation in local guidelines between centres, these patients may not have been fully investigated at initial presentation and therefore would have had a delay in diagnosis. This work demonstrates that a negative faecal calprotectin does not always reassuringly exclude IBD. That if low FC is used to decide not to investigate further, it should continue to be monitored if patients are symptomatic. It is not known whether a low FC at diagnosis represents an early stage of disease. We now aim to look at disease progression for our low FC group to investigate whether starting management at this point delays need for escalation of treatment.

\section{P07 A SINGLE-CENTRE PROSPECTIVE OBSERVATIONAL STUDY COMPARING PROACTIVE WITH REACTIVE THERAPEUTIC DRUG MONITORING (TDM) IN TWO COHORTS OF CHILDREN WITH INFLAMMATORY BOWEL DISEASE (IBD)}

Marco Gasparetto, Natasha Burgess, Dominic Studart, Sandhia Naik, Ahmed Kadir, Nick Croft, lan Sanderson, Protima Deb. The Royal London Children's Hospital, Barts Health Trust, Department of Paediatric Gastroenterology

\subsection{6/flgastro-2021-bspghan.18}

Background Primary non-response (PNR) and secondary loss of response (LoR) to anti-TNF therapy are a significant challenge in up to $45 \%$ of patients with IBD. Therapeutic drug monitoring (TDM) refers to the practice of measuring antiTNF trough level and anti-drug antibody to guide clinical
Abstract P06 Figure 5 In patients with Ulcerative, there was a significant difference in FC (Faecal calprotectin) values between the normal/low FC and raised/high FC groups, ${ }^{* * *} \mathrm{p}<0.0001$ (MannWhitney test) 